\title{
How to get rid of one of the weights in a two-weight Poincaré inequality?
}

\author{
by Bruno Franchi (Bologna) and Piotr HajŁasz (Warszawa)
}

To the memory of Bogdan Ziemian

Abstract. We prove that if a Poincaré inequality with two different weights holds on every ball, then a Poincaré inequality with the same weight on both sides holds as well.

Introduction. By a two-weight Poincaré inequality we mean an inequality of the form

$$
\left(f_{B}\left|u-u_{B, \nu}\right|^{p} d \nu\right)^{1 / p} \leq C r\left(f_{B}|\nabla u|^{p} d \mu\right)^{1 / p},
$$

which is supposed to hold for every ball $B \subset \mathbb{R}^{n}$ of radius $r$ and every $u \in C^{\infty}(B)$. Here, $1 \leq p<\infty, \mu$ and $\nu$ are two positive Radon measures, and the following notation is used for the average:

$$
u_{B, \nu}=f_{B} u d \nu=\nu(B)^{-1} \int_{B} u d \nu .
$$

The aim of this paper is to prove that, if the above Poincaré inequality holds on every ball and for every smooth function $u$, then we can replace $\nu$ by $\mu$ on the left hand side. For a precise statement, see Corollary 1 below. In fact, our present result can be viewed in the more general context of self-improving properties of two-weight Poincaré inequalities that will be studied in [15]; nevertheless, we present here this direct proof because of its simplicity.

2000 Mathematics Subject Classification: Primary 46E35.

Key words and phrases: Poincaré inequality, weights, metric spaces, doubling measures.

B.F. was partially supported by Italian MURST and GNAFA of CNR, Italy, and P.H. by KBN grant no. 2-PO3A-055-14. The research was carried out during the stay of P.H. at the Max-Planck Institute for Mathematics in the Sciences in Leipzig, 1998. He thanks the Institute for support and hospitality. 
Our main result, Theorem 1, is stated in the very general setting of Poincaré inequalities on metric spaces endowed with doubling measures. The theory of Sobolev spaces on metric spaces was basically originated in [20]. Dealing with such a general situation is justified by numerous applications to Sobolev inequalities on Riemannian manifolds, analysis on graphs, and Sobolev spaces associated with vector fields: see e.g. [10] and [22], where the reader will find an exhaustive bibliography on the subject.

Let $(X, d)$ be a metric space. We say that a Borel measure $\mu$ on $(X, d)$ is doubling if there is a constant $C_{\mu}>0$ such that for every ball $B \subset X$, $\mu(2 B) \leq C_{\mu} \mu(B)$. In addition we require that $\mu(B)>0$ on every ball $B \subset X$ and that $\mu$ is finite on all bounded sets.

Here and in the sequel we denote by $\lambda B, \lambda>0$, the ball concentric with $B$ and with radius $\lambda$ times that of $B$. By $L_{\text {loc }}^{p}(\mu)$ we denote the class of functions which are $L^{p}$ integrable with respect to $\mu$ on every ball. By $C$ we denote a general positive constant; its value may change even in a single string of estimates.

We would like to thank Dick Wheeden for many stimulating and fruitful discussions, and for having generously shared his ideas with us. We also thank Jan Malý for a fruitful discussion. The idea we earned from him helped us simplify our original proof.

Main result. The main result of the paper reads as follows.

Theorem 1. Let $(X, d)$ be a metric space endowed with two doubling measures $\mu$ and $\nu$, where $\mu$ is absolutely continuous with respect to $\nu$. Let $u \in L_{\mathrm{loc}}^{p}(\nu)$ and $0 \leq g \in L_{\mathrm{loc}}^{p}(\mu), p \geq 1$. Assume that on every ball $B \subset X$ the following version of the "two-weight Poincaré inequality" holds:

$$
\left(f_{B}\left|u-u_{B, \nu}\right|^{p} d \nu\right)^{1 / p} \leq C r\left(f_{\sigma B} g^{p} d \mu\right)^{1 / p},
$$

where $r$ is the radius of the ball $B$ and $C>0, \sigma \geq 1$ are fixed constants independent of $B$. Then there is another constant $C^{\prime}>0$ such that on every ball $B \subset X$ we have the following "one-weight" inequality:

$$
\left(f_{B}\left|u-u_{B, \mu}\right|^{p} d \mu\right)^{1 / p} \leq C^{\prime} r\left(f_{5 \sigma B} g^{p} d \mu\right)^{1 / p} .
$$

Remarks. 1) If we replace the local integrability of $u$ by a stronger condition that $u$ is continuous and bounded on every ball, then we do not have to assume that $\mu$ is absolutely continuous with respect to $\nu$.

2) Actually the same proof yields slightly more. Indeed, instead of (2) it suffices to assume a weaker inequality 


$$
f_{B}\left|u-u_{B, \nu}\right| d \nu \leq C r\left(f_{\sigma B} g^{p} d \mu\right)^{1 / p},
$$

and the conclusion remains the same.

3) The functions $u$ and $g$ are counterparts of a Sobolev function and the absolute value of its gradient. One can prove that Poincaré type inequalities like those in Theorem 1 imply that the function $u$ inherits most of the properties of classical Sobolev functions, as we can see in the references cited above in connection with the Sobolev spaces on metric spaces.

Proof of Theorem 1. Let $x \in B$ be a Lebesgue point of $u$ with respect to the measure $\nu$. Put $B_{i}(x)=B\left(x, 2^{i}\right)$. Let $i_{0}$ be the least integer such that $2^{i_{0}} \geq \operatorname{diam} B$. Then $B \subset B_{i_{0}}(x)$. Since, by the Lebesgue differentiation theorem [22, Theorem 14.15], $u_{B_{i}, \nu} \rightarrow u(x)$ as $i \rightarrow-\infty$ for $\nu$-a.e. $x \in X$, we conclude that

$$
\begin{aligned}
\left|u(x)-u_{B, \nu}\right| & \leq\left|u_{B, \nu}-u_{B_{i_{0}}, \nu}\right|+\sum_{i=-\infty}^{i_{0}}\left|u_{B_{i}, \nu}-u_{B_{i-1}, \nu}\right| \\
& \leq \frac{\nu\left(B_{i_{0}}\right)}{\nu(B)} f_{B_{i_{0}}}\left|u-u_{B_{i_{0}}, \nu}\right| d \nu+\sum_{i=-\infty}^{i_{0}} \frac{\nu\left(B_{i}\right)}{\nu\left(B_{i-1}\right)} f_{B_{i}}\left|u-u_{B_{i}, \nu}\right| d \nu \\
& \leq C \sum_{i=-\infty}^{i_{0}} f_{B_{i}}\left|u-u_{B_{i}, \nu}\right| d \nu \\
& \leq C \sum_{i=-\infty}^{i_{0}} 2^{i}\left(f_{\sigma B_{i}} g^{p} d \mu\right)^{1 / p} \\
& \leq C\left(\sum_{i=-\infty}^{i_{0}} 2^{i}\right)^{1-1 / p}\left(\sum_{i=-\infty}^{i_{0}} 2^{i} f g^{p} d \mu\right)^{1 / p} \\
& \leq C r^{1-1 / p}\left(\sum_{i=-\infty}^{i_{0}} 2^{i} f_{\sigma B_{i}} g^{p} d \mu\right)^{1 / p} .
\end{aligned}
$$

Hence

$$
f_{B}\left|u-u_{B, \nu}\right|^{p} d \mu \leq C r^{p-1} \sum_{i=-\infty}^{i_{0}} 2^{i} f_{B} f_{\sigma B_{i}} g^{p} d \mu d \mu=\diamond .
$$

Applying the doubling property of the measure $\mu$ and Fubini's theorem yields

$$
f_{B} f_{\sigma B_{i}} g^{p} d \mu d \mu=f_{B} \int_{X} \frac{\chi_{B\left(x, 2^{i} \sigma\right)}(z)}{\mu\left(B\left(x, 2^{i} \sigma\right)\right)} g^{p}(z) d \mu(z) d \mu(x)
$$




$$
\begin{aligned}
& \leq C \int_{X} \int_{B} \frac{\chi_{B\left(z, 2^{i} \sigma\right)}(x)}{\mu\left(B\left(z, 2^{i} \sigma\right)\right)} d \mu(x) g^{p}(z) d \mu(z) \\
& \leq C \int_{X} \frac{\chi_{5 \sigma B}(z)}{\mu(B)} g^{p}(z) d \mu(z) \leq C \int_{5 \sigma B} g^{p} d \mu .
\end{aligned}
$$

Hence we can continue:

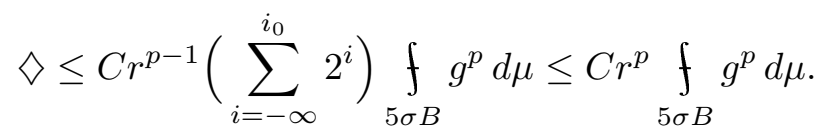

Now the elementary inequality

$$
\left(f_{B}\left|u-u_{B, \mu}\right|^{p} d \mu\right)^{1 / p} \leq 2\left(f_{B}\left|u-u_{B, \nu}\right|^{p} d \mu\right)^{1 / p}
$$

completes the proof of the theorem.

REMARK. The absolute continuity of $\mu$ with respect to $\nu$ was employed to deduce that $\mu$-almost all points $x \in B$ are Lebesgue points for $u$ with respect to the measure $\nu$. If we assume that $u$ is continuous, then all points are Lebesgue points and hence we do not need to require that $\mu$ is absolutely continuous with respect to $\nu$.

The above result directly applies to two-weight Poincaré inequalities in $\mathbb{R}^{n}$.

Corollary 1. Let $\mu$ and $\nu$ be two positive Radon measures in $\mathbb{R}^{n}$ satisfying the doubling condition. Let $1 \leq p<\infty$ and $C>0$ be fixed constants. Assume that

$$
\left(f_{B}\left|u-u_{B, \nu}\right|^{p} d \nu\right)^{1 / p} \leq C r\left(f_{B}|\nabla u|^{p} d \mu\right)^{1 / p},
$$

whenever $B$ is a ball and $u \in C^{\infty}(B)$ (here $r$ is the radius of $B$ ). Then there is another constant $C^{\prime}>0$ such that

$$
\left(f_{B}\left|u-u_{B, \mu}\right|^{p} d \mu\right)^{1 / p} \leq C^{\prime} r\left(f_{B}|\nabla u|^{p} d \mu\right)^{1 / p},
$$

for all balls $B$ and all $u \in C^{\infty}(B)$.

Proof. Theorem 1 together with the remark gives the result with the ball $5 B$ on the right hand side. Now the fact that we can replace $5 B$ by $B$ is standard; see for example [21] for a short proof. The proof is complete.

Remarks. 1) Corollary 1 can be easily generalized to the setting of Poincaré inequalities associated with vector fields, as those in [23], [6], [8][11], [13], [19], [22] and the recent [25]. 
2) The method that allows one to reduce the size of the ball on the right hand side is well known. It seems there are at least three basic techniques to do that: the one developed by Boman, [4], [3], [5], [7], [9], [19], [26], [27]; the one developed by Kohn, [24], [23]; and the one recently discovered in [21] and [22].

3) So far, we have proved a result for Poincaré inequalities such as (3). We call them $(p, p)$-inequalities, because of the presence of the same exponent $p$ on both sides. One could ask whether it is possible to get a similar result for $(p, q)$-Sobolev-Poincaré inequalities, with a larger exponent $q$ on the left-hand side. In fact, such a generalization can be easily obtained. Indeed, it has recently been discovered that the $(p, p)$-Poincaré inequality like $(3)$ enjoys the so-called self-improving property, i.e. it implies the $(p, q)$-SobolevPoincaré inequality with the optimal exponent on the left hand side: see [29], [1], [2], [13], [14], [19], [21], [22], [28], so that there is no loss of generality in taking the same exponent $p$ on both sides of (3).

Suppose $\mu$ is the Lebesgue measure in $\mathbb{R}^{n}$, and let $X=\left(X_{1}, \ldots, X_{m}\right)$ be a system of Lipschitz continuous vector fields in $\mathbb{R}^{n}$. If $\lambda$ belongs to the class $A_{\infty}$ of Fefferman-Stein and Muckenhoupt for the Carnot-Carathéodory metric, then $d \mu=d x$ is absolutely continuous with respect to $d \nu=\lambda(x) d x$, and both measures are doubling (cf. [18, IV.2]). We denote by $u_{B, \lambda}$ and $u_{B}$ the average values of $u$ over $B$ with respect to the measures $\lambda(x) d x$ and $d x$ respectively.

Now, if $u$ is a continuously differentiable function, we denote by $|X u|^{2}=$ $\sum_{j}\left|X_{j} u\right|^{2}$ the gradient associated with $X$. With these notations, the following result is an easy consequence of Theorem 1 .

Corollary 2. Suppose $\lambda \in A_{\infty}$ with respect to the Carnot-Carathéodory metric associated with $X$. If there are constants $C>0$ and $\sigma \geq 1$ such that for any Carnot-Carathéodory ball $B=B(x, r)$ and any continuously differentiable function $u$ we have

$$
\left(f_{B}\left|u-u_{B, \lambda}\right|^{p} \lambda(x) d x\right)^{1 / p} \leq C r\left(f_{\sigma B}|X u|^{p} d x\right)^{1 / p}
$$

then (with a new choice of the constant $C$ )

$$
\left(f_{B}\left|u-u_{B}\right|^{p} d x\right)^{1 / p} \leq \operatorname{Cr}\left(f_{B}|X u|^{p} d x\right)^{1 / p} .
$$

Indeed, in this case we can get rid of the enlarging factor $5 \sigma$ that appears in (3) by a Boman chain type argument as in [9].

The interest of the above example originates from the following situation: suppose there exists a compensation couple $(\lambda, s)$ for $X\left(\lambda \in L_{\mathrm{loc}}^{1}, s>1\right)$ in 
the sense of [17], i.e. there exist constants $c, C>0$ such that

$$
c(\mu(B) / r)^{s} \leq \int_{B} \lambda(y) d y \leq C(\mu(B) / r)^{s}
$$

for any Carnot-Carathéodory ball $B=B(x, r)$. In fact, the theory in [17] is developed for smooth vector fields; nevertheless, the definition of compensation couple and the arguments we develop require only the Lipschitz continuity of the vector fields. If $\lambda$ belongs to the class $A_{\infty}$ (as it happens e.g. for smooth vector fields satisfying Hörmander's rank condition), then Corollary 2 above holds.

\section{References}

[1] D. Bakry, T. Coulhon, M. Ledoux and L. Saloff-Coste, Sobolev inequalities in disguise, Indiana Univ. Math. J. 44 (1995), 1033-1074.

[2] M. Biroli and U. Mosco, Sobolev inequalities on homogeneous spaces, Potential Anal. 4 (1995), 311-324.

[3] B. Bojarski, Remarks on Sobolev imbedding inequalities, in: Complex Analysis (Joensuu, 1987), Lecture Notes in Math. 1351, Springer 1988, 52-68.

[4] J. Boman, $L^{p}$-estimates for very strongly elliptic systems, report no 29, Dept. of Math., Univ. of Stockholm, 1982.

[5] S. M. Buckley, P. Koskela and G. Lu, Boman equals John, in: XVI Rolf Nevanlinna Colloquium (Joensuu,1995), de Gruyter, Berlin, 1996, 91-99.

[6] L. Capogna, D. Danielli and N. Garofalo, Subelliptic mollifiers and a basic pointwise estimate of Poincaré type, Math. Z. 226 (1997), 147-154.

[7] S. K. Chua, Weighted Sobolev inequalities on domains satisfying the chain condition, Proc. Amer. Math. Soc. 117 (1993), 449-457.

[8] B. Franchi, Weighted Sobolev-Poincaré inequalities and pointwise inequalities for a class of degenerate elliptic equations, Trans. Amer. Math. Soc. 327 (1991), 125158.

[9] B. Franchi, C. E. Gutiérrez and R. L. Wheeden, Weighted Sobolev-Poincaré inequalities for Grushin type operators, Comm. Partial Differential Equations 19 (1994), 523-604.

[10] B. Franchi, P. Hajłasz and P. Koskela, Definitions of Sobolev classes on metric spaces, Ann. Inst. Fourier (Grenoble) 49 (1999), 1903-1924.

[11] B. Franchi and E. Lanconelli, Hölder regularity theorem for a class of linear nonuniformly elliptic operators with measurable coefficients, Ann. Scuola Norm. Sup. Pisa 10 (1983), 523-541.

[12] B. Franchi, G. Lu and R. L. Wheeden, Representation formulas and weighted Poincaré inequalities for Hörmander vector fields, Ann. Inst. Fourier (Grenoble) 45 (1995), 577-604.

[13] - - - - A relationship between Poincaré-type inequalities and representation formulas in spaces of homogeneous type, Internat. Mat. Res. Notices 1996, no. 1, 1-14.

[14] B. Franchi, C. Pérez and R. L. Wheeden, Self-improving properties of JohnNirenberg and Poincaré inequalities on spaces of homogeneous type, J. Funct. Anal. 153 (1998), 108-146.

[15] - , - - , in preparation. 
[16] B. Franchi and R. L. Wheeden, Some remarks about Poincaré type inequalities and representation formulas in metric spaces of homogeneous type, J. Inequalities Appl. 3 (1999), 65-89

[17] - - - Compensation couples and isoperimetric estimates for vector fields, Colloq. Math. 74 (1997), 1-27.

[18] J. García Cuerva and J. Rubio de Francia, Weighted Norm Inequalities and Related Topics, North-Holland, Math. Stud. 116, North-Holland, 1985.

[19] N. Garofalo and D. M. Nhieu, Isoperimetric and Sobolev inequalities for CarnotCarathéodory spaces and the existence of minimal surfaces, Comm. Pure Appl. Math. 49 (1996), 1081-1144.

[20] P. Hajłasz, Sobolev spaces on an arbitrary metric space, Potential Anal. 5 (1996), 403-415.

[21] P. Hajłasz and P. Koskela, Sobolev meets Poincaré, C. R. Acad. Sci. Paris 320 (1995), 1211-1215.

[22] —, -, Sobolev met Poincaré, Mem. Amer. Math. Soc. 145 (2000).

[23] D. Jerison, The Poincaré inequality for vector fields satisfying Hörmander's condition, Duke Math. J. 53 (1986), 503-523.

[24] R. V. Kohn, New integral estimates for the deformations in terms of their nonlinear strains, Arch. Rational Mech. Anal. 78 (1982), 131-172.

[25] E. Lanconelli and D. Morbidelli, On the Poincaré inequality for vector fields, Ark. Mat. 38 (2000), 327-342.

[26] G. Lu, Weighted Poincaré and Sobolev inequalities for vector fields satisfying Hörmander's condition and applications, Rev. Mat. Iberoamericana 8 (1992), 367439.

[27] - The sharp Poincaré inequality for free vector fields: an endpoint result, ibid. 10 (1994), 453-466.

[28] P. Maheux et L. Saloff-Coste, Analyse sur les boules d'un opérateur souselliptique, Math. Ann. 303 (1995), 713-740.

[29] L. Saloff-Coste, A note on Poincaré, Sobolev, and Harnack inequalities, Internat. Mat. Res. Notices 1992, no. 2, 27-38.

Dipartimento di Matematica

Università di Bologna

Piazza di Porta S. Donato, 5

I-40127 Bologna, Italy

E-mail: franchib@dm.unibo.it
Institute of Mathematics

Warsaw University

Banacha 2

02-097 Warszawa, Poland

E-mail: hajlasz@mimuw.edu.pl

Reçu par la Rédaction le 30.4.1999

Révisé le 20.7.1999 